\title{
Array-based satellite phase bias sensing: theory and GPS/BeiDou/QZSS results
}

\author{
A Khodabandeh ${ }^{1}$ and P J G Teunissen ${ }^{1,2}$ \\ 1 Department of Spatial Sciences, Curtin University of Technology, Perth, Australia \\ 2 Department of Geoscience and Remote Sensing, Delft University of Technology, \\ Delft, The Netherlands \\ E-mail: amir.khodabandeh@curtin.edu.au \\ E-mail: p.teunissen@curtin.edu.au
}

\begin{abstract}
Single-receiver integer ambiguity resolution (IAR) is a measurement concept that makes use of network-derived non-integer satellite phase biases (SPBs), among other corrections, to recover and resolve the integer ambiguities of the carrierphase data of a single GNSS receiver. If it is realized, the very precise integer ambiguity-resolved carrier-phase data would then contribute to the estimation of the receiver's position, thus making (near) real-time precise point positioning feasible. Proper definition and determination of the SPBs take a leading part in developing the idea of single-receiver IAR. In this contribution, the concept of array-based betweensatellite single-differenced (SD) SPB determination is introduced which is aimed to reduce the code-dominated precision of the SD-SPB corrections. The underlying model is realized by giving the role of the local reference network to an array of antennas, mounted on rigid platforms, that are separated by short distances so that the same ionospheric delay is assumed to be experienced by all the antennas. To that end, a closed-form expression of the array-aided SD-SPB corrections is presented, thereby proposing a simple strategy to compute the SD-SPBs. After resolving doubledifferenced ambiguities of the array's data, the variance of the SD-SPB corrections is shown to be reduced by a factor equal to the number of antennas. This improvement in precision is also affirmed by numerical results of the three GNSSs GPS, BeiDou and QZSS. Experimental results demonstrate that the integer-recovered ambiguities converge to integers faster, upon increasing the number of antennas aiding the SD-SPB corrections.
\end{abstract}

Keywords: GNSS, precise point positioning (PPP), single-receiver integer ambiguity resolution (IAR), single-differenced satellite phase bias (SD-SPB)

Submitted to: Meas. Sci. Technol.

\section{Introduction}

Precise point positioning (PPP) of a single GNSS receiver has found a widespread usage in various Geoscience and atmospheric disciplines [1, 2]. The technique relies 
on the carrier-phase and code data of a single receiver, profiting from network-derived corrections for satellite orbits, clocks and (sometimes) atmospheric delays [3, 4]. Taking advantage of the millimeter-level precision of the carrier-phase data, the aim is to precisely position the location of a user. Since the carrier-phase data are biased by the real-valued (but time-constant) ambiguities, they would, however, not take a truly active part in estimating the user's position, unless a rather long convergence time takes placelonger than 30 min to attain sub-decimeter level of accuracy [5, 6]. Fast and centimeter level PPP would therefore get feasible, if the ambiguities are successfully resolved to integer values. Unfortunately, because of the presence of the satellite hardware biases in the carrier-phase and code data, the estimable between-satellite single-differenced (SD) ambiguities of the PPP user are not of an integer nature. In other words, the integer-valued ambiguities cannot be separated from these biases.

Recent contributions have demonstrated that the stated satellite hardware biases can be properly defined and estimated as the estimable satellite phase biases (also referred to as uncalibrated phase delays or fractional cycle biases), see e.g. [7, 8, 9, 10, $11,12,13]$. Given a-priori network-derived SPBs, the integer property of the user's SD ambiguities is thus recovered, giving rise to single-receiver integer ambiguity resolution (IAR). In this respect, the estimable SPBs are either presented in their undifferenced form $[14,15,16]$, undifferenced between-frequency-combined form [17, 18], betweensatellite SD form [19], between-satellite SD between-frequency-combined form [8, 12], or assimilated into the clocks defining decoupled phase clock parameters $[9,10,13]$.

As shown in the contributions by $[16,19]$, the precision of the SPB corrections is governed by the code noise. Although significantly shortening the convergence time, PPP ambiguity resolution does therefore still take about 15 min of time to reliably fix the ambiguities to their integers, would no ionospheric corrections be applied [20].

In this paper, the concept of array-based between-satellite SD-SPB determination is introduced. We convey the idea of SPB determination to the case where the role of the local reference network is taken by an array of antennas, mounted on rigid platforms, that are separated by short distances so that the same ionospheric delay is assumed to be experienced by all the antennas [21]. The objective of this study is to develop an efficient strategy to reduce the code-dominated noise of the SD-SPB corrections, expediting the convergence of the user's integer-recovered ambiguities over time. To that end, closed-form analytical expressions of the SD-SPB corrections are presented and the dependency of SD-SPB precision on the number of aiding antennas is analyzed. Upon resolving double-differenced (DD) ambiguities, the code-dominated variance of the SD-SPB corrections is shown to be reduced by a factor equal to the number of antennas. Thus the larger the number of antennas are utilized, the more precise the array-aided SD-SPB corrections become.

The paper is organized as follows. In section 2, a full-rank model of the singleantenna observation equations is presented. We show that the estimable SD-SPB is, in fact, a combination of the true SD-SPB, the SD satellite code hardware delay, and the SD ambiguity of a reference antenna. Analytical SD-SPB estimators are presented in section 
3. We commence with the corrections provided by a single antenna. The insensitivity of the single-antenna SD-SPB corrections to the dispersive and non-dispersive effects is pointed out. In particular, we show that the single-antenna SD-SPB corrections are nothing else, but linear functions of the code multipath equations. The contribution of the aiding antennas to the precision of the SD-SPB corrections is then formulated. It is shown that including the data of the aiding antennas results in the same number of unknowns, i.e. the DD ambiguities. Provided that no further information is available, the contribution of the aiding antennas would thus not make any sense unless the DD ambiguities are successfully resolved. Presenting a closed-form expression of the arrayaided SD-SPB corrections, a simple algorithm to compute them is outlined afterwards. Finally in section 4, the performance of the proposed methodology is validated by a GPS/BeiDou/QZSS campaign collecting dual-frequency data.

We make use of the following notation: Random variables are distinguished by an underscore. Thus $\underline{x}$ is random, while $x$ is not. The expectation of $\underline{x}$ is denoted by $\mathrm{E}\{\underline{x}\}$, while its standard deviation is denoted by $\sigma_{x}$. The identity matrix and the summation vector (vector of ones) are, respectively, denoted as $I$ and $e$, where their dimension is

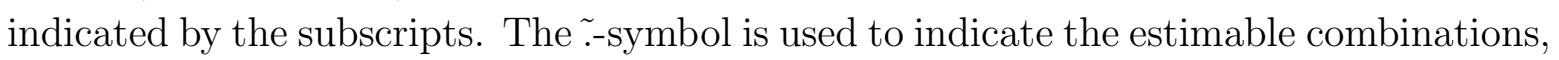
while the --symbol and -symbol are used to indicate the estimators before and after IAR, respectively. The antenna, satellite and frequency indexes are, respectively, denoted by $r=1, \ldots, n ; s=1, \ldots, m$ and $j=1,2$. Throughout the text, we make use of the Kronecker matrix product $\otimes$. For its properties, we refer to, e.g., [22, 23].

\section{Single-antenna full-rank model}

Consider a reference antenna, say $r=1$, tracking dual-frequency GNSS data of the rover satellite $s$, given the pivot satellite $s=1$. The between-satellite SD observation equations of the satellite pairs $1 s(s=2, \ldots, m)$ read then [24]

$$
\begin{aligned}
& \mathrm{E}\left\{\underline{\phi}_{1, j}^{1 s}\right\}=\rho_{1}^{1 s}-\mu_{j} \iota_{1}^{1 s}+\lambda_{j}\left(a_{1, j}^{1 s}-\delta_{, j}^{1 s}\right) \\
& \mathrm{E}\left\{\underline{p}_{1, j}^{1 s}\right\}=\rho_{1}^{1 s}+\mu_{j} \iota_{1}^{1 s}-d_{, j}^{1 s}
\end{aligned}
$$

where $\underline{\phi}_{1, j}^{1 s}$ and $\underline{p}_{1, j}^{1 s}$ denote the between-satellite SD phase and code observables on frequency $j(j=1,2)$, respectively. The non-dispersive parameter $\rho_{1}^{1 s}$ captures the SD geometric ranges, SD tropospheric delays, as well as the SD satellite clock parameters. The (first-order) SD slant ionospheric delay, experienced on the first frequency, is denoted by $\iota_{1}^{1 s}$. Expressed in cycles, the SD ambiguity is composed of the integervalued parameter $a_{1, j}^{1 s}$ and the $\mathrm{SD}-\mathrm{SPB} \delta_{, j}^{1 s}$ which are linked to the phase observables through the wavelength $\lambda_{j}$. Likewise, the SD satellite code hardware delays are denoted by $d_{, j}^{1 s}$. The frequency-dependent coefficients are defined as the ratio $\mu_{j}=\left(\lambda_{j}^{2} / \lambda_{1}^{2}\right)$. We remark, apart from $a_{1, j}^{1 s}$ and $\delta_{, j}^{1 s}$, that the rest of the quantities have been expressed in units of range. 
Multivariate representation In the remainder of this paper, we make of use the more compact multivariate notation, just for the sake of presentation. In doing so, we define the SD phase observation vector as $\underline{\phi}_{1}=\left[\underline{\phi}_{1,1}^{T}, \underline{\phi}_{1,2}^{T}\right]^{T}$, with $\underline{\phi}_{1, j}(j=1,2)$ being a vector, of size $m-1$, which contains $\underline{\phi}_{1, j}^{1 s}(s=2, \ldots, m)$. The SD code observations vector $\underline{p}_{1}$ is defined similarly. With these in mind, the multivariate version of (1) can be stated as

$$
\begin{aligned}
& \mathrm{E}\left\{\underline{\phi}_{1}\right\}=\left[e_{2} \otimes I_{m-1}\right] \rho_{1}-\left[\mu \otimes I_{m-1}\right] \iota_{1}+\left[\Lambda \otimes I_{m-1}\right]\left(a_{1}-\delta\right) \\
& \mathrm{E}\left\{\underline{p}_{1}\right\}=\left[e_{2} \otimes I_{m-1}\right] \rho_{1}+\left[\mu \otimes I_{m-1}\right] \iota_{1}-d
\end{aligned}
$$

where the vectors $\rho_{1}$ and $\iota_{1}$, of size $m-1$, contain $\rho_{1}^{1 s}$ and $\iota_{1}^{1 s}(s=2, \ldots, m)$, respectively. The $2 \times 2$ diagonal matrix $\Lambda$ contains the wavelengths $\lambda_{j}(j=1,2)$, while the frequencydependent vector is defined as $\mu=\left[\mu_{1}, \mu_{2}\right]^{T}$. The SD ambiguity vector is defined as $a_{1}=\left[a_{1,1}^{T}, a_{1,2}\right]^{T}$, with $a_{1, j}(j=1,2)$ being a vector, of size $m-1$, which contains $a_{1, j}^{1 s}$ $(s=2, \ldots, m)$. The vectors of the SD-SPBs $\delta$ and the SD satellite code hardware delays $d$ are defined similarly.

The observation equations, given in (2), do not represent a full-rank model, in the sense that the parameters involved cannot be separated from one another. It is therefore only possible to estimate combinations of the parameters. These estimable combinations are formed according to the minimum set of parameters which need to be lumped with the remaining parameters so that the underlying rank-deficiency is eliminated [11]. This minimum set of parameters is referred to as an $\mathcal{S}$-basis of the model defining a minimum number of constraints through which the model becomes of full-rank [25, 26].

As shown in $[16,19]$, the code hardware delays $d$ and the integer-valued ambiguities $a_{1}$ can, for instance, be chosen as the $\mathcal{S}$-basis of the model. In doing so, we define the ionosphere-free (IF) and geometry-free (GF) combinations of $d$, respectively, as

$$
\begin{aligned}
& d_{I F}=\left[\mu_{I F}^{T} \otimes I_{m-1}\right] d, \quad \text { with } \quad \mu_{I F}=\frac{1}{\mu_{12}}\left[\mu_{2},-\mu_{1}\right]^{T} \\
& d_{G F}=\left[\mu_{G F}^{T} \otimes I_{m-1}\right] d, \quad \text { with } \quad \mu_{G F}=\frac{1}{\mu_{12}}[-1,1]^{T}
\end{aligned}
$$

where $\mu_{12}=\mu_{2}-\mu_{1}$.

On the basis of the above definition, the original code hardware delays stand in one-to-one correspondence with their IF and GF counterparts through

$$
d=\left[e_{2} \otimes I_{m-1}\right] d_{I F}+\left[\mu \otimes I_{m-1}\right] d_{G F},
$$

since $e_{2} \mu_{I F}^{T}+\mu \mu_{G F}^{T}=I_{2}$.

With the aid of the representation (4), it is evident that the IF component $d_{I F}$ is absorbed by the non-dispersive vector $\rho_{1}$, while the GF component $d_{G F}$ is absorbed by the ionospheric delays $\iota_{1}$. This yields the following estimable combinations

$$
\tilde{\rho}_{1}=\rho_{1}-d_{I F}, \quad \tilde{\iota}_{1}=\iota_{1}-d_{G F}
$$

Substituting $\rho_{1}=\tilde{\rho}_{1}+d_{I F}$ and $\iota_{1}=\tilde{\iota}_{1}+d_{G F}$ into (2), a full-rank model is then structured by lumping $a_{1}$ with $\delta$, that is

$$
\begin{aligned}
& \mathrm{E}\left\{\underline{\phi}_{1}\right\}=\left[e_{2} \otimes I_{m-1}\right] \tilde{\rho}_{1}-\left[\mu \otimes I_{m-1}\right] \tilde{\iota}_{1}-\left[\Lambda \otimes I_{m-1}\right] \tilde{\delta} \\
& \mathrm{E}\left\{\underline{p}_{1}\right\}=\left[e_{2} \otimes I_{m-1}\right] \tilde{\rho}_{1}+\left[\mu \otimes I_{m-1}\right] \tilde{\iota}_{1},
\end{aligned}
$$


where the estimable SD-SPBs take the following form

$$
\tilde{\delta}=\delta-L_{p} d-a_{1}, \quad \text { with } \quad L_{p}=\Lambda^{-1}\left(e_{2} \mu_{I F}^{T}-\mu \mu_{G F}^{T}\right) \otimes I_{m-1}
$$

Therefore, the estimable SD-SPBs are composed of the true SD-SPBs $\delta$, the SD satellite code hardware delays $d$ and the SD ambiguities of the reference antenna $a_{1}$. When expressed in units of range rather than in cycles, the above estimable SD-SPBs become indeed identical to those presented in [19].

\section{Analytical solution to the SD-SPB corrections}

\subsection{Single-antenna $S D-S P B$ corrections}

The number of observations in (6) is as many as the number of estimable parameters. In particular, the number of $\tilde{\delta}$ is equal to that of $\underline{\phi}_{1}$ and, at the same time, the estimable $\mathrm{SD}-\mathrm{SPBs}$ are not present in the code observables. The phase observables are thus fully reserved to determine the estimable SD-SPBs. As a consequence, the estimable parameters $\tilde{\rho}_{1}$ and $\tilde{\iota}_{1}$ are, respectively, determined by the IF and GF combinations of the code observables only, i.e.

$$
\underline{\tilde{\tilde{\rho}}}_{1}=\left[\mu_{I F}^{T} \otimes I_{m-1}\right] \underline{p}_{1}, \quad \underline{\tilde{\tilde{}}}_{1}=\left[\mu_{G F}^{T} \otimes I_{m-1}\right] \underline{p}_{1},
$$

Given the above code-based estimators, the single-antenna estimator of the estimable SD-SPBs follows from the first expression of (6), namely (Appendix)

$$
\underline{\tilde{\tilde{\delta}}}=L_{p} \underline{p}_{1}-L_{\phi} \underline{\phi}_{1}, \quad \text { with } \quad L_{\phi}=\Lambda^{-1} \otimes I_{m-1}
$$

Thus, in addition to the phase observables, the single-antenna SD-SPB estimator is a linear function of the IF and GF code combinations as well.

Relation to the code multipath equations According to (6), any non-dispersive bias, equally experienced by both the code and phase observations, would be absorbed by the non-dispersive parameter $\tilde{\rho}_{1}$. Likewise, any dispersive bias, equally experienced by both the code and phase observations, would be absorbed by the dispersive parameter $\tilde{\iota}_{1}$. This, namely, shows that the SD-SPB estimator is insensitive to any non-dispersive and dispersive unmodeled effects. In case unwanted effects only affect, for instance, the code observations however, the outcome of the SD-SPB estimator would change. Of these types of effects, the code multipath is well-known. A traditional diagnostic tool for tracing possible code multipath effects is to check the time-stability of the so-called multipath equations [27]. Using the expressions $L_{p}$ and $L_{\phi}$, respectively, given in (7) and (9), the code multipath equations can be expressed as [28, p. 224]

$$
\underline{\eta}=\underline{p}_{1}-L_{\phi}^{-1} L_{p} \underline{\phi}_{1}
$$

It is not difficult to verify the identity $L_{p} L_{\phi}^{-1} L_{p}=L_{\phi}$. With this mind, pre-multiplying the preceding equation by $L_{p}$, together with (9), establishes a one-to-one relationship between the SD-SPB estimator and the code multipath as follows

$$
\underline{\hat{\tilde{\delta}}}=L_{p} \underline{\eta} \Longleftrightarrow \underline{\eta}=L_{p}^{-1} \underline{\tilde{\tilde{\delta}}}
$$



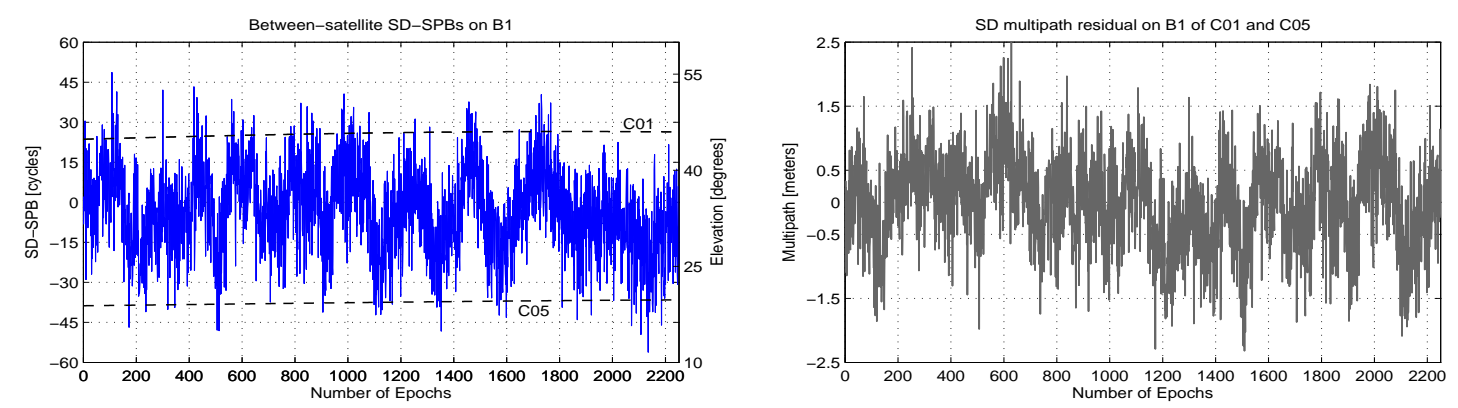

Figure 1. Left: SD-SPB corrections on B1 $\left(\underline{\tilde{\tilde{\delta}}}_{B_{1}}\right)$ corresponding to BeiDou geostationary satellites $\mathrm{C} 01$ and $\mathrm{C} 05$ over time. Satellites' elevation is depicted in black dashed line. Right: The associated SD multipath combinations $\left(\underline{\eta}_{, B_{1}}\right)$.

This indeed shows that the SD-SPB corrections are nothing else but linear functions of the code multipath equations. Fig. 1 illustrates the SD-SPB corrections on $B_{1}$ of BeiDou geostationary satellites $\mathrm{C} 01$ and $\mathrm{C} 05$ as well as the corresponding SD multipath combinations over time. The adverse effect of the multipath, i.e. the periodic behavior, is clearly observed in both the SD-SPB corrections and the multipath combinations. In other words, the SD-SPB corrections can be directly affected by the code multipath.

From single-epoch estimator to multi-epoch estimator The estimator, given in (9), is based on data of one single epoch, i.e. the SD-SPBs are assumed to be unlinked in time. It is therefore referred to as the single-epoch estimator. In cases where information on the temporal behavior of the parameters, in (2), are assumed given, a joint estimation procedure of the estimable parameters, over time, must be applied [14]. In this study, we only assume the SD-SPBs $\delta$, the SD satellite code hardware delays $d$, along with the SD ambiguities $a_{1}$ to behave constant over time. The rest of the parameters are considered to be unlinked in time. Upon these assumptions, the multi-epoch estimator of the estimable SD-SPBs follows by weighted averaging its single-epoch counterparts over epochs, that is

$$
\underline{\tilde{\tilde{\delta}}}[k]=\frac{1}{k} \sum_{i=1}^{k}\left[I_{2} \otimes \bar{W}_{[k]}^{-1} W_{i}\right] \underline{\tilde{\tilde{\delta}}}(i), \quad \text { with } \quad \bar{W}_{[k]}=\frac{1}{k} \sum_{i=1}^{k} W_{i}
$$

where $\underline{\tilde{\delta}}[k]$ denotes the multi-epoch estimator of the SD-SPBs over $k$ epochs. The single-epoch estimator of the SD-SPBs, based on the data of epoch $i$, is denoted by $\underline{\tilde{\tilde{\delta}}}(i)$. The $(m-1) \times(m-1)$ diagonal weight matrices $W_{i}(i=1, \ldots, k)$ contain the nonnegative coefficients $w_{i}^{1 s}(s=2, \ldots, m)$ capturing the dependency of the GNSS data on the satellites' elevation. Here we make use of the exponential elevation weighting strategy [29]. They are, namely, computed as

$$
w_{i}^{1 s}=\left(\frac{1}{w_{i}^{1}}+\frac{1}{w_{i}^{s}}\right)^{-1}
$$

with

$$
w_{i}^{s}=\left[1+10 \exp \left(-\frac{\epsilon_{i}^{s}}{10^{\circ}}\right)\right]^{-2}, \quad s=1, \ldots, m
$$




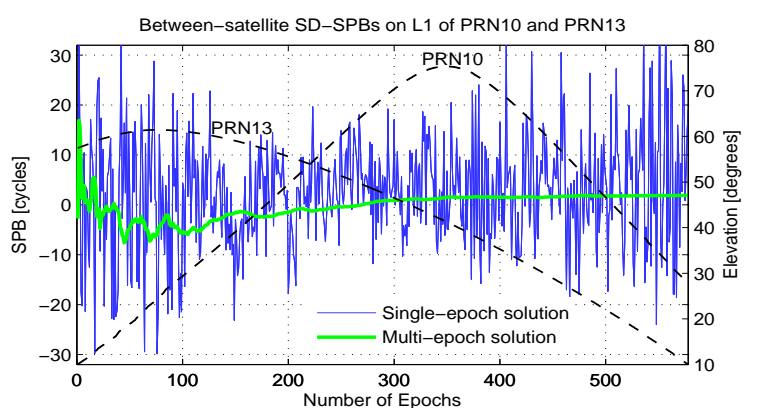

Figure 2. GPS single-epoch SD-SPB corrections $\underline{\tilde{\tilde{\delta}}}_{L_{1}}(i)$ (in blue) compared to its multi-epoch counterparts $\underline{\tilde{\tilde{\delta}}}_{L_{1}}[k]$ (in green) over time. Satellites' elevation is depicted in black dashed line.

where $\epsilon_{i}^{s}$ is the elevation of satellite $s$ [degree] at epoch $i$ with respect to the reference antenna.

Fig. 2 shows typical examples of the single-epoch and multi-epoch versions of the SD-SPB corrections. It is observed that the size of the fluctuations of the single-epoch corrections increases as the elevation of the satellites decreases, showing the elevation dependency of the SD-SPB corrections. As the number of epochs increases, the multiepoch SD-SPB corrections get more stable in time and converge to a constant value.

From non-combined $S D$-SPBs to combined $S D$-SPBs The closed-form expression of (9) enables us to analyze the precision of the single-epoch single-antenna SD-SPB estimators. The precision analysis can be conducted for those on the original frequencies (i.e. the non-combined SD-SPBs) as well as for those on a linear combinations of the frequencies (i.e. the combined SD-SPBs). Consider GPS dual-frequency data where the code observables on both the frequencies are assumed to be uncorrelated and equally precise. Uncorrelated with the code data, the phase observables are also assumed to be uncorrelated and equally precise. Let $\sigma_{p}$ and $\sigma_{\phi}=0.01 \sigma_{p}$ be the standard deviations of the between-satellite SD code and phase observables in meters, respectively. Using the coefficients $\lambda_{j}$ and $\mu_{j}$ of GPS/BeiDou systems as given in Table 1, an application of the error propagation law to $(9)$ gives

$$
\begin{aligned}
\sigma_{\tilde{\tilde{\delta}}_{, L 1}} & \approx 26.95 \sigma_{p}[\text { cycle }], \quad \sigma_{\tilde{\tilde{\delta}}_{, L 2}} \approx 26.75 \sigma_{p}[\text { cycle }] \\
\sigma_{\tilde{\tilde{\delta}}_{, B 1}} & \approx 25.85 \sigma_{p}[\text { cycle }], \quad \sigma_{\tilde{\tilde{\delta}}_{, B 2}} \approx 25.64 \sigma_{p}[\text { cycle }]
\end{aligned}
$$

Table 1. GPS/BeiDou wavelengths $\lambda_{j}$ and frequency-dependent coefficients $\mu_{j}$ (Dualfrequency scenario).

\begin{tabular}{lcccc}
\hline & L1 & L2 & B1 & B2 \\
\hline$\lambda_{j}(\mathrm{~cm})$ & 19.03 & 24.42 & 19.20 & 24.83 \\
$\mu_{j}$ & 1 & 1.6467 & 1 & 1.6724 \\
\hline
\end{tabular}



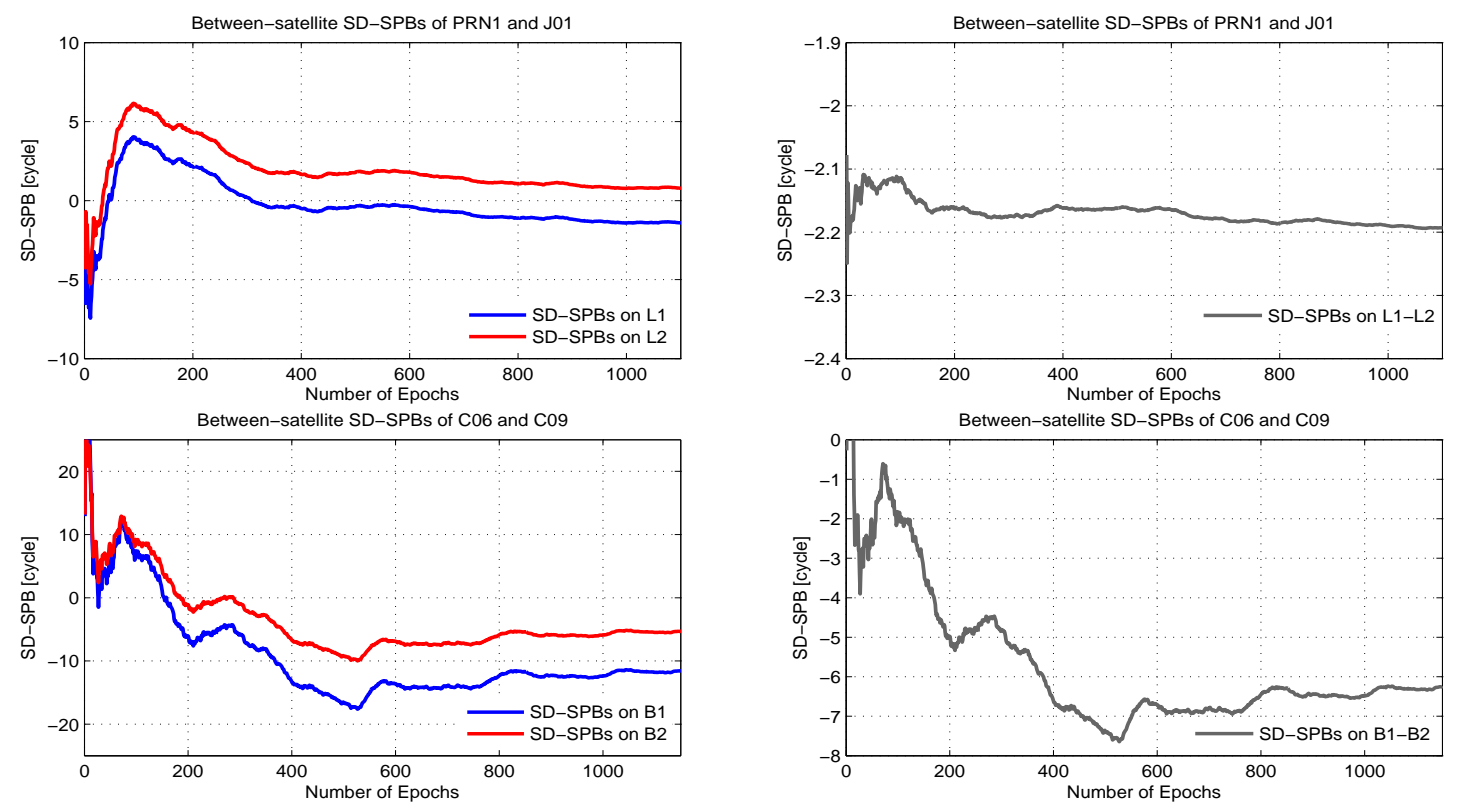

Figure 3. Top-left: Multi-epoch SD-SPB corrections on L1 (in blue) and on L2 (in red) of GPS. Top-right: Multi-epoch SD-SPB corrections on L1-L2, i.e. wide-lane SD-SPB corrections (in gray) of GPS. Bottom-left: Multi-epoch SD-SPB corrections on B1 (in blue) and on B2 (in red) of BeiDou. Bottom-right: Multi-epoch SD-SPB corrections on B1-B2, i.e. wide-lane SD-SPB corrections (in gray) of BeiDou.

Thus the precision of the non-combined SD-SPBs is heavily dominated by the noise of the code data. The error propagation law also provides us with the cross-correlation between the SD-SPBs on L1/B1 and L2/B2. In case of GPS, the stated correlation is about 0.9996, whereas in case of BeiDou, the correlation is about 0.9918. As shown in Fig. 3 (left-panel), the signatures of the SD-SPB corrections on both the frequencies are almost the same, corroborating that the SD-SPBs on L1/B1 and L2/B2 are highly correlated.

Of the combined SD-SPBs, one can, for instance, consider the wide-lane SD-SPBs that are defined as the between-frequency difference of the non-combined SD-SPBs (i.e. those on L1-L2 and on B1-B2). Given the standard deviations of the non-combined SD-SPBs (cf. (15)), together with their cross-correlation, the standard deviation of the wide-lane SD-SPBs is obtained as

$$
\left.\sigma_{\tilde{\tilde{\delta}}_{, L 1-L 2}} \approx 0.83 \sigma_{p}[\text { cycle }], \quad \sigma_{\hat{\tilde{\delta}}_{, B 1-B 2}} \approx 0.84 \sigma_{p} \text { [cycle }\right]
$$

which is roughly 32 times smaller than those of the non-combined SD-SPBs. As shown in Fig. 3 (right-panel), the size of the variations of the SD-SPB corrections on L1-L2 is much smaller than that of their non-combined counterparts.

\subsection{Array-aided SD-SPB corrections}

To reduce the code-dominated noise of the SD-SPB corrections (cf. (15)), one may think of including the data of another antenna, say $r(r \neq 1)$, for which a similar full-rank 
model can be formed by replacing subscript 1 with $r$ in (6). As shown in (7), our earlier estimable SD-SPBs depend on the ambiguities of the reference antenna $a_{1}$. The new full-rank model would, however, offer another form of the estimable SD-SPBs dependent on $a_{r}$ instead, that is

$$
\tilde{\tilde{\delta}}=\delta-L_{p} d-a_{r}
$$

where their corresponding estimator reads (cf. (9))

$$
\underline{\tilde{\tilde{\delta}}}=L_{p} \underline{p}_{r}-L_{\phi} \underline{\phi}_{r}
$$

Comparing (17) with (7), these new estimable SD-SPBs are related to $\tilde{\delta}$ through

$$
\tilde{\tilde{\delta}}=\tilde{\delta}-a_{1 r},
$$

with the DD ambiguities $a_{1 r}=a_{r}-a_{1}$.

According to (19), including the data of antenna $r$ results in $2(m-1)$ additional equations $\underline{\tilde{\tilde{\tilde{\delta}}}}$, given in $(18)$, at the expense of $2(m-1)$ unknown DD ambiguities $a_{1 r}$. Therefore, as long as the DD ambiguities are unresolved, the extra antenna $r$ has no contribution to the determination of the SD-SPBs $\tilde{\delta}$.

Let us, for the moment, assume $a_{1 r}$ to be successfully fixed to their integers $\check{a}_{1 r}$. With (9) and (18), we then arrive at the following equations

$$
\begin{aligned}
& \mathrm{E}\{\underline{\hat{\tilde{\delta}}}\} \\
& \mathrm{E}\{\underline{\tilde{\tilde{\delta}}}\}+\check{a}_{1 r}=\tilde{\delta}
\end{aligned}
$$

in which the first expression characterizes the contribution of the reference antenna $r=1$, while the second expression characterizes that of the extra antenna $r \neq 1$. Assuming the receivers/antennas of the same type, one can simply consider the average of the above two sets of equations as the improved estimator of the SD-SPB corrections $\tilde{\delta}$. The idea can be generalized to the case where the data of $n-1$ extra antennas $r=2, \ldots, n$ are incorporated into the model. Given the resolved DD ambiguities, the array-aided SD-SPB estimator $\underline{\tilde{\tilde{\delta}}}$ reads then

$$
\underline{\tilde{\tilde{\delta}}}=L_{p} \underline{p}_{\bar{r}}-L_{\phi} \underline{\phi}_{\bar{r}}+\check{a}_{1 \bar{r}}
$$

in which the following quantities are introduced

$$
\underline{p}_{\bar{r}}=\frac{1}{n} \sum_{r=1}^{n} \underline{p}_{r}, \quad \underline{\phi}_{\bar{r}}=\frac{1}{n} \sum_{r=1}^{n} \underline{\phi}_{r}, \quad \check{a}_{1 \bar{r}}=\frac{1}{n} \sum_{r=1}^{n} \check{a}_{1 r}
$$

with $\check{a}_{11}=0$.

Array-based ambiguity-fixing As stated before, the data of the extra antennas do not contribute to the determination of the SD-SPBs, would the DD ambiguities remain unresolved. This is why one needs the average of the resolved ambiguities $\check{a}_{1 r}(r=$ $1, \ldots, n)$ to form the array-aided SD-SPB estimator in (21).

Although GNSS ambiguity-fixing is generally a bit involved, the procedure gets, however, considerably easier if simplified assumptions are placed on the model. Here, we 
consider an array of antennas, separated by short distances, so that the same ionospheric delay is assumed to be experienced by all the antennas [21], that is $\iota_{1 r}=\iota_{r}-\iota_{1} \approx 0$ $(r=2, \ldots, n)$. Given information on the geometry of the satellites/antennas, the DD geometric ranges are computed, namely $\underline{\hat{\rho}}_{1 r}=\underline{\hat{\rho}}_{r}-\underline{\hat{\rho}}_{1}(r=2, \ldots, n)$. The float DD ambiguities are then obtained as follows [30, p. 390]

$$
\underline{\hat{a}}_{1 r}=L_{\phi}\left\{\underline{\phi}_{1 r}-\left[e_{2} \otimes I_{m-1}\right] \underline{\hat{\rho}}_{1 r}\right\}, \quad r=2, \ldots, n
$$

with $\underline{\hat{a}}_{1 r}$ and $\underline{\phi}_{1 r}=\underline{\phi}_{r}-\underline{\phi}_{1}$ being the DD float ambiguities and phase observables, respectively. Having successfully resolved the DD float ambiguities, the algorithmic steps in computing the array-aided SD-SPB corrections have been summarized in Fig. 4.

\subsection{Superiority of the array-aided $S D-S P B$ corrections}

Superiority in precision Since the array-aided SD-SPB estimator $\underline{\tilde{\tilde{\delta}}}$ is structured by the average of the data of $n$ antennas, its variance is $n$ times smaller than that of the singleantenna estimator $\underline{\tilde{\tilde{\delta}}}$, provided that the data of all the antennas are equally precise. Thus their standard deviations are related to one another through

$$
\sigma_{\tilde{\delta}_{, j}}=\frac{1}{\sqrt{n}} \sigma_{\tilde{\tilde{\delta}}_{, j}}, \quad j=1,2
$$

Superiority in expediting the convergence of the user's ambiguities As a direct consequence of better precision of the array-aided SD-SPB estimator as compared to its single-antenna counterpart, the integer-recovered user ambiguities converge to their integers faster, would they be corrected by the array-aided SD-SPB corrections. Let $k_{n}$ be the minimum number of epochs at which the user's ambiguity of the satellite pair

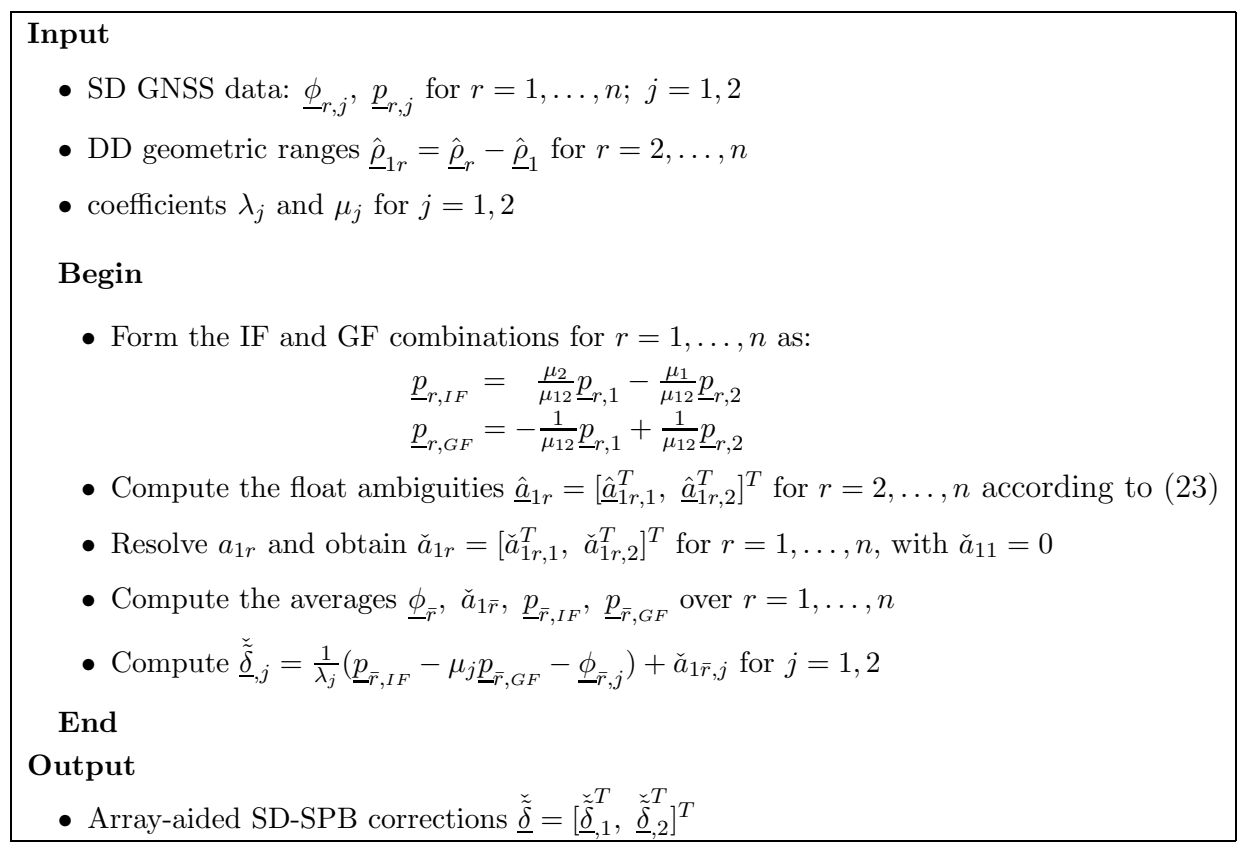

Figure 4. Algorithmic steps in computing the array-aided SD-SPBs. 

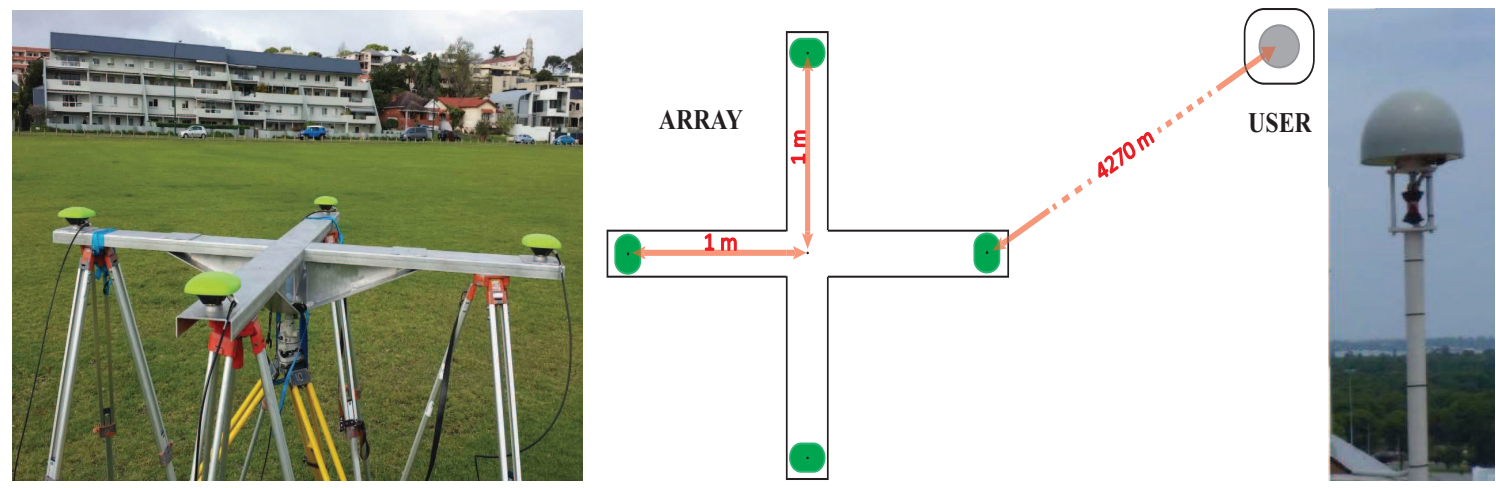

Figure 5. Structure of the array of antennas (left) and the user (right) as used in the experiment. The distance between the user's antenna and the reference antenna of the array is about 4270 meters.

$1 s$ on frequency $j$, recovered by the array-aided SD-SPBs, meets certain precision. The ratio of $k_{n}$ to its corresponding single-antenna version, say $k_{1}$, can be formulated as (Appendix)

$$
\frac{k_{n}}{k_{1}}=\frac{n \alpha+1}{n(1+\alpha)} \frac{\bar{w}_{\left[k_{1}\right]}^{1 s}}{\bar{w}_{\left[k_{n}\right]}^{1 s}}, \quad \text { with } \quad \bar{w}_{\left[k_{n}\right]}^{1 s}=\frac{1}{k_{n}} \sum_{i=1}^{k_{n}} w_{i}^{1 s}
$$

where the scalar $\alpha$ denotes the variance-ratio of the data of the user antenna to those of the array's reference antenna.

The preceding equation does in fact show that the reduction in the convergence time $1-\left(k_{n} / k_{1}\right)$ depends on the three contributing factors, namely, 1) the elevation of the satellite pair $1 s$ characterized by $\left.\left(\bar{w}_{\left[k_{1}\right]}^{1 s} / \bar{w}_{\left[k_{n}\right]}^{1 s}\right), 2\right)$ the quality of the user data with respect to the array data characterized by $\alpha$, and 3) the number of aiding antennas $n$. As a special case where no elevation weighting is applied (i.e. $\bar{w}_{\left[k_{n}\right]}^{1 s}=\bar{w}_{\left[k_{1}\right]}^{1 s}$ ) and the user receiver/antenna is of the same type as the array receiver/antenna (i.e. $\alpha=1$ ), the equation is simplified to

$$
\frac{k_{n}}{k_{1}}=\frac{n+1}{2 n} \quad \text { or } \quad 1-\frac{k_{n}}{k_{1}}=\frac{n-1}{2 n}
$$

Since $(n-1) /(2 n)$ is a monotonically increasing function of $n$, the more the number antennas are employed, the larger the reduction in the convergence time $1-\left(k_{n} / k_{1}\right)$ (thus the faster the convergence time) becomes. In the case where the number of antennas $n$ is large enough, the convergence time can therefore be reduced up to 50 percent. We remark that the expression (25) is restricted to the ambiguity convergence on a single frequency. The convergence does indeed improve, would information about the ambiguities on both frequencies be considered. 
Table 2. RMSE of the single-epoch SD-SPB corrections corresponding to GPS PRN10 and PRN13 for different number of antennas. The RMSE ratio has been compared to its theoretical value $(1 / \sqrt{n})$.

\begin{tabular}{l|c|c|c|c|c|c|c|c} 
No. of antennas & \multicolumn{2}{|c|}{$n=1$} & \multicolumn{2}{c|}{$n=2$} & \multicolumn{2}{c|}{$n=3$} & \multicolumn{2}{c}{$n=4$} \\
\hline & L1 & L1-L2 & L1 & L1-L2 & L1 & L1-L2 & L1 & L1-L2 \\
\hline RMSE [cycle] & 10.77 & 0.36 & 7.88 & 0.25 & 6.42 & 0.22 & 5.71 & 0.19 \\
\hline RMSE ratio & 1.00 & 1.00 & 0.73 & 0.69 & 0.60 & 0.61 & 0.53 & 0.53 \\
\hline Theoretical value & 1.00 & 1.00 & 0.71 & 0.71 & 0.58 & 0.58 & 0.50 & 0.50 \\
\hline
\end{tabular}

\section{Numerical results}

\subsection{Experiment description}

In this section, the methodology discussed above is evaluated by an array-based experiment organized by Curtin University in Perth, Australia on 12 September 2013. Two GPS/BeiDou dual-frequency data-sets have been collected by 5 GNSS receivers: 1) the array data-set of 4 Javad receivers (TRE-G3T Delta) of the same antennas and 2) the user data-set of 1 Trimble receiver (NETR9). The frequencies considered are L1 and L2 (transmitted by GPS) as well as B1 and B2 (transmitted by BeiDou). In one case, the L1/L2 data of the single QZSS satellite J01 is also considered. The structure of the array and user has been visualized in Fig. 5. The array was located in Foreshore, South Perth, whereas the user station was set up at Bentley campus of Curtin University. The distance between the reference antenna of the array and the user antenna is about 4 $[\mathrm{km}]$. All the data have been measured above a cut-off elevation of $10^{\circ}$ with a sampling interval of 10 seconds during 00:00:00-11:32:00 in GPS time. The array data-set is aimed to determine the SD-SPB corrections, while the user data-set is aimed to show the applicability of the stated corrections.

\subsection{SD-SPB's precision improvement}

We first consider the results of the array data-set. To gain some insight into the dependency of the SD-SPBs' precision on the number of antennas, time-series of the single-epoch SD-SPB estimator have been presented. It is clear that the size of the fluctuations of each time-series gives an impression of the noise of the corresponding SD-SPB corrections. Shown in Fig. 6 are the time-series of the SD-SPB corrections as a function of the number of antennas. The results correspond to QZSS satellite J01, GPS satellites PRN1, PRN10, PRN13 and BeiDou satellites C06, C10. In all these cases, the noise reduction can be observed as the number of antennas increases from $n=1$ (red line) to $n=4$ (green line).

To quantify the noise of the single-epoch SD-SPB corrections, we make use of the root mean squared error (RMSE) of the time-series. The RMSE of the single-epoch array-aided SD-SPBs of GPS and BeiDou are, respectively, given in Tables 2 and 3. As shown, for instance, the RMSE of the single-antenna SD-SPBs on L1 is computed as 

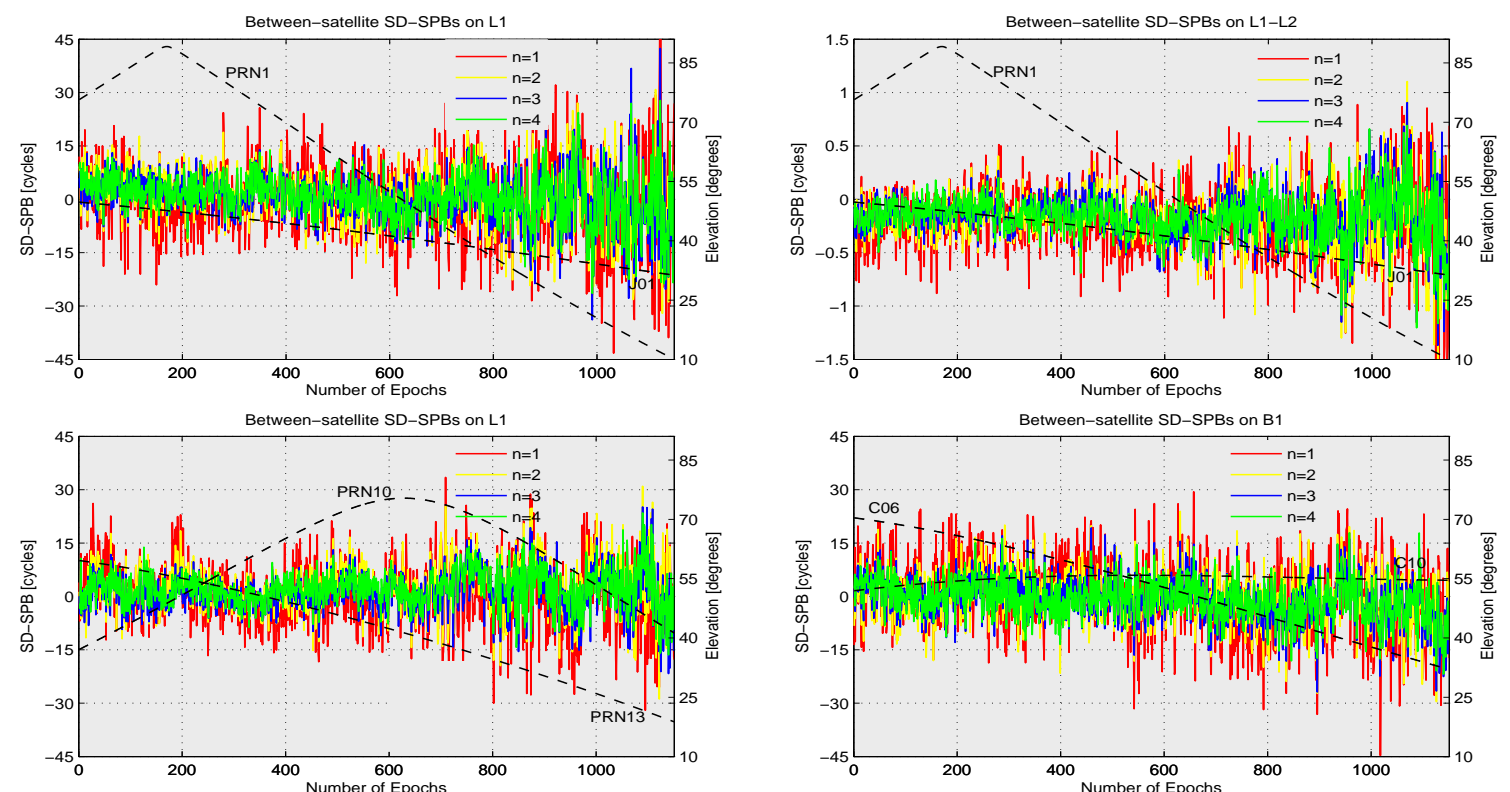

Figure 6. Single-epoch SD-SPB corrections over time as a function of the number of antennas $(n)$; top-left: between QZSS and GPS PRN1 on L1; top-right: between QZSS and GPS PRN1 on L1-L2; bottom-left: between GPS PRN10 and PRN13 on L1; bottom-right: between BeiDou C06 and C10 on B1.

10.77 [cycle] which is almost 32 times larger than that of the SD-SPBs on L1-L2 (0.36 [cycle]). This is in agreement with the theoretical value already inferred from (15) and (16). According to (24), one would expect the ratio of the RMSE of the array-aided SD-SPBs to that of the single-antenna SD-SPBs to be approximated by $(1 / \sqrt{n})$. The RMSE ratios have been compared to their theoretical counterparts in Tables 2 and 3 . As shown, the RMSE ratios and the theoretical values, to a large extent, coincide. In case of GPS, the RMSE of the single-epoch SD-SPBs on L1 decreases from 10.77 [cycle] to almost half its value, i.e. 5.71 [cycle]. In case of BeiDou, a similar scenario occurs, i.e. from 12.05 [cycle] to 6.53 [cycle].

Table 3. RMSE of the single-epoch SD-SPB corrections corresponding to BeiDou C06 and $\mathrm{C} 10$ for different number of antennas. The RMSE ratio has been compared to its theoretical value $(1 / \sqrt{n})$.

\begin{tabular}{l|c|c|c|c|c|c|c|c} 
No. of antennas & \multicolumn{2}{|c|}{$n=1$} & \multicolumn{2}{c|}{$n=2$} & \multicolumn{2}{c|}{$n=3$} & \multicolumn{2}{c}{$n=4$} \\
\hline & B1 & B1-B2 & B1 & B1-B2 & B1 & B1-B2 & B1 & B1-B2 \\
\hline RMSE [cycle] & 12.05 & 0.43 & 8.87 & 0.31 & 7.41 & 0.26 & 6.53 & 0.23 \\
\hline RMSE ratio & 1.00 & 1.00 & 0.74 & 0.72 & 0.61 & 0.60 & 0.54 & 0.53 \\
\hline Theoretical value & 1.00 & 1.00 & 0.71 & 0.71 & 0.58 & 0.58 & 0.50 & 0.50 \\
\hline
\end{tabular}



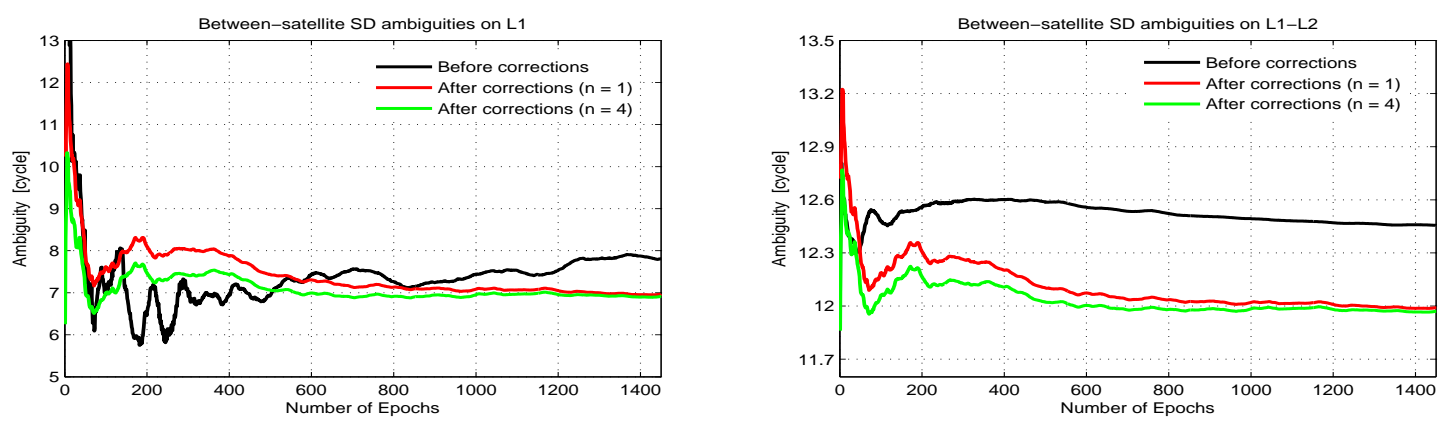

Figure 7. Convergence of the user's SD ambiguities on L1 (left) and on L1-L2 (right) of GPS over time. The results correspond to PRN10, given PRN13 as the pivot satellite.

\subsection{Integer recovery of the user's ambiguities}

We now turn our attention to the results of the user data-set. Forming the betweensatellite SD combinations of the user's observations, the receiver-specific unknown parameters are removed. Like the array data-set, the satellite positions have been computed using the orbits of the IGS. The estimable between-satellite SD satellite clocks and ionospheric delays have been estimated using the array data-set and sent to the user according to the strategy as described in $[16,19]$. Since the distance between the array and the user is about $4[\mathrm{~km}]$, correcting the user's data by the same ionospheric delays, estimated at the array's location, is realistically allowed. Corrected by the array-derived satellite clocks and ionospheric delays, the SD observation equations of the user only include the position and real-valued SD ambiguities as unknowns.

We now evaluate the integer recovery role of the SD-SPB corrections. To do so, we consider the convergence of the user's SD ambiguities over time, once without applying the SD-SPB corrections, and another time with applying the SD-SPB corrections. The corresponding results have been illustrated in Fig. 7. It is clearly visible that the SD ambiguities, without corrections, do not tend to integer values. Corrected by the SD$\mathrm{SPBs}$ however, the integer property of the SD ambiguities is recovered and they do indeed converge to integer values.

In view of the SD-SPBs' precision improvement discussed earlier, one would expect that the array-aided SD-SPBs expedite the ambiguities' convergence compared to the single-antenna SD-SPBs. This has been realized in Fig. 7 where the time-series of the multi-epoch ambiguities, aided by the corrections of 4 antennas (green lines), goes faster to integers than its counterpart aided by 1 antenna (red lines). More precisely, one can measure the minimum number of epochs $k_{n}$ through which the precision of the estimated ambiguities reaches a certain value, e.g. 0.1 [cycle], thus computing the reduction in the convergence time $1-\left(k_{n} / k_{1}\right)$. This observed reduction can also be predicted by the rule given in (25). Both the observed and predicted outcomes have been provided in Table 4. In case of GPS, the variance-ratio is taken as $\alpha=1.3$, while, in case of BeiDou, it is taken as $\alpha=1.1$. In either case, the convergence time reduces as the number of antennas increases. The rates of the the reduction in the convergence time 
Table 4. Reduction in the convergence time of the integer-recovered SD ambiguities $1-\left(k_{n} / k_{1}\right)$ (percent). The observed results have been computed based on the minimum number of epochs at which the precision of the ambiguities reaches 0.1 [cycle]. The predicted values have been computed using (25).

\begin{tabular}{l|cc|cc|cc|c}
\multicolumn{1}{c|}{ No. of antennas } & \multicolumn{2}{|c|}{$n=2$} & \multicolumn{2}{c|}{$n=3$} & \multicolumn{2}{c|}{$n=4$} & $n=10$ \\
\hline & observed & predicted & observed & predicted & observed & predicted & predicted \\
\hline GPS: PRN10-PRN13 (L1) & 9.5 & 9.0 & 13.1 & 12.3 & 15.3 & 15.8 & 23.9 \\
\hline BeiDou: C06-C10 (B1) & 15.5 & 17.8 & 24.6 & 23.5 & 27.6 & 26.1 & 34.3 \\
\hline
\end{tabular}

are different though. In case of BeiDou, the reduction in time is considerable, while in case of GPS, a smaller reduction in time is observed. The reasoning is as follows. As shown in the bottom-panel of Fig. 6, the elevation of PRN13 of GPS rapidly decreases over time. This makes the elevation-dependent weight $\bar{w}_{\left[k_{n}\right]}^{1 s}$ get smaller as the number of epochs increases. Given the ratio $\left(\bar{w}_{\left[k_{1}\right]}^{1 s} / \bar{w}_{\left[k_{4}\right]}^{1 s}\right) \approx 1.25>1$ in the numerator of $(25)$, the minimum number of epochs $k_{4}$ is therefore not significantly smaller than $k_{1}$. In case of C06 and C10 of BeiDou however, the weight ratio is smaller than that of the GPS scenario, i.e. $\left(\bar{w}_{\left[k_{1}\right]}^{1 s} / \bar{w}_{\left[k_{4}\right]}^{1 s}\right) \approx 1.14$, meaning that the reduction in time is mainly governed by the number of antennas $n$.

It is worth highlighting that the rule, given in (25), enables us to predict the reduction in the convergence time for a larger number of aiding antennas $n$. For instance, would we make use of $n=10$ number of antennas, the reduction becomes about 24 percent for the GPS case, and about 34 percent for the BeiDou case, assuming $\left(\bar{w}_{\left[k_{1}\right]}^{1 s} / \bar{w}_{\left[k_{4}\right]}^{1 s}\right)=\left(\bar{w}_{\left[k_{1}\right]}^{1 s} / \bar{w}_{\left[k_{10}\right]}^{1 s}\right)($ cf. Table 4$)$.

\section{Conclusions}

In this contribution, we introduced the concept of array-based between-satellite SD-SPB determination - the key parameter to perform single-receiver IAR. The underlying model is characterized by data of an array of GNSS antennas, mounted on rigid platforms, that are separated by short distances so that the same ionospheric delay is assumed to be experienced by all the antennas. Forming a full-rank GNSS model, the estimable SDSPBs were shown to appear, for instance, as a combination of the true SD-SPBs, the $\mathrm{SD}$ satellite code hardware delays, and the SD ambiguities of a reference antenna.

A closed-form expression of the array-aided SD-SPB corrections was presented through which a simple strategy to compute the SD-SPBs was outlined. The link between the SD-SPB corrections and the code multipath equations was established. As linear combinations of the multipath equations, the SD-SPB corrections can be directly affected by the unwanted effects of the multipath. Therefore, essential care needs to be taken in locating reference stations processing SPB corrections.

In order to reduce the code-dominated variance of the SD-SPB corrections, the data of extra antennas are incorporated into the model. As shown, the extra antennas give additional information on the SD-SPBs at the expense of additional unknowns, i.e. 
the array's DD ambiguities. After resolving the DD ambiguities of the array's data, the extra antennas do contribute to the precision of the SD-SPBs. With $n$ being the number of all contributing antennas, the variance of the array-aided SD-SPB corrections is $n$ times smaller than that of the single-antenna SD-SPB corrections. This precision improvement was also demonstrated by numerical results. As a consequence of which, the integer-recovered ambiguities of the user converge to integer values faster, upon increasing the number of aiding antennas.

\section{Acknowledgments}

The array-based experiment was conducted by Dr. N. Raziq and Dr. B. Padovan from Curtin University, Perth, Australia. This support is gratefully acknowledged.

\section{Appendix A.}

Proof of (9) As the number of observations in (6) is as many as the number of estimable parameters, the SD-SPB solution follows from

$$
\left[\Lambda \otimes I_{m-1}\right] \underline{\tilde{\tilde{\delta}}}=-\underline{\phi}_{1}+\left[e_{2} \otimes I_{m-1}\right] \underline{\tilde{\tilde{\rho}}}_{1}-\left[\mu \otimes I_{m-1}\right] \underline{\tilde{\tilde{\underline{u}}}}_{1}
$$

With regard to (8), pre-multiplying the preceding equation by $\Lambda^{-1} \otimes I_{m-1}$ gives (9).

Proof of (25) Let $\sigma_{a}^{2}$ be the variance of the multi-epoch solution of the ambiguity of the satellite pair $1 s$ on frequency $j$. It is obtained as

$$
\sigma_{a}^{2}=\sigma_{n}^{2} \frac{1}{k_{n} \bar{w}_{\left[k_{n}\right]}^{1 s}}
$$

where $\sigma_{n}^{2}$ is the variance of the corresponding single-epoch ambiguity solution, aided by the corrections of an array of $n$ antennas. According to (A.2), the number of epochs, needed to achieve the variance $\sigma_{a}^{2}$, follows as

$$
k_{n}=\frac{\sigma_{n}^{2}}{\sigma_{a}^{2}} \frac{1}{\bar{w}_{\left[k_{n}\right]}^{1 s}} \quad \text { thus } \quad \frac{k_{n}}{k_{1}}=\frac{\sigma_{n}^{2}}{\sigma_{1}^{2}} \frac{\bar{w}_{\left[k_{1}\right]}^{1 s}}{\bar{w}_{\left[k_{n}\right]}^{1 s}}
$$

The equality (25) is therefore proven, if we demonstrate

$$
\frac{\sigma_{n}^{2}}{\sigma_{1}^{2}}=\frac{n \alpha+1}{n(1+\alpha)}
$$

Since, by definition, $\alpha$ is the variance-ratio of the data of the user antenna to that of the array's reference antenna, say $\sigma_{y}^{2}$, one can obtain

$$
\sigma_{n}^{2}=\alpha \sigma_{y}^{2}+\frac{1}{n} \sigma_{y}^{2}=\frac{1+n \alpha}{n} \sigma_{y}^{2}
$$

The first term $\alpha \sigma_{y}^{2}$ is the uncertainty due to the user data, while the second term $(1 / n) \sigma_{y}^{2}$ is the uncertainty due to the array-aided SD-SPB corrections. This proves (A.4). 


\section{References}

[1] P. Heroux and J. Kouba, "GPS precise point positioning with a difference," in Paper presented at Geomatics 95, Ottawa, Canada, pages 13-15, 1995.

[2] J. F. Zumberge, M. B. Heflin, D. C. Jefferson, M. M. Watkins, and F. H. Webb, "Precise point positioning for the efficient and robust analysis of GPS data from large networks," J. Geophys. Res., vol. 102, pages 5005-5017, 1997.

[3] P. Wielgosz, S. Cellmer, Z. Rzepecka, J. Paziewski, and D. Grejner-Brzezinska, "Troposphere modeling for precise GPS rapid static positioning in mountainous areas," Meas. Sci. Technol., vol. 22 , no. 4, p. 045101, 2011.

[4] T. Hadas, J. Kaplon, J. Bosy, J. Sierny, and K. Wilgan, "Near-real-time regional troposphere models for the GNSS precise point positioning technique," Meas. Sci. Technol., vol. 24, no. 5, p. 055003, 2013.

[5] R. F. Leandro, M. C. Santos, and R. B. Langley, "Analyzing GNSS data in precise point positioning software," GPS Solut., vol. 15, no. 1, pages 1-13, 2011.

[6] R. J. van Bree and C. C. Tiberius, "Real-time single-frequency precise point positioning: accuracy assessment," GPS Solut., vol. 16, no. 2, pages 259-266, 2012.

[7] M. J. Gabor and R. S. Nerem, "Satellite-satellite single-difference phase bias calibration as applied to ambiguity resolution," Navigation, vol. 49, no. 4, pages 223-242, 2002.

[8] M. Ge, G. Gendt, M. Rothacher, C. Shi, and J. Liu, "Resolution of GPS carrier-phase ambiguities in precise point positioning (PPP) with daily observations," J. Geod., vol. 82, no. 7, pages 389399, 2008.

[9] D. Laurichesse, F. Mercier, J. Berthias, P. Broca, L. Cerri, and F. CNES, "Integer ambiguity resolution on undifferenced GPS phase measurements and its application to PPP and satellite precise orbit determination," Navigation, vol. 56, no. 2, pages 135-149, 2009.

[10] P. Collins, S. Bisnath, F. Lahaye, and P. Heroux, "Undifferenced GPS ambiguity resolution using the decoupled clock model and ambiguity datum fixing," Navigation, vol. 57, no. 2, pages 123$135,2010$.

[11] P. J. G. Teunissen, D. Odijk, and B. Zhang, "PPP-RTK: Results of CORS Network-Based PPP with Integer Ambiguity Resolution," J. Aeronaut. Astronaut. Aviat., vol. 42, no. 4, pages 223$229,2010$.

[12] J. Geng, C. Shi, M. Ge, A. H. Dodson, Y. Lou, Q. Zhao, and J. Liu, "Improving the estimation of fractional-cycle biases for ambiguity resolution in precise point positioning," J. Geod., vol. 86, no. 8, pages 579-589, 2012.

[13] S. Loyer, F. Perosanz, F. Mercier, H. Capdeville, and J.-C. Marty, "Zero-difference GPS ambiguity resolution at CNES-CLS IGS Analysis Center," J. Geod., vol. 86, no. 11, pages 991-1003, 2012.

[14] P. Henkel, Z. Wen, and C. Gunther, "Estimation of satellite and receiver biases on multiple Galileo frequencies with a Kalman filter," in ION Proceedings of the 2010 International Technical Meeting of The Institute of Navigation, CA, pages 1067-1074, 2010.

[15] Z. Wen, P. Henkel, and C. Gunther, "Reliable estimation of phase biases of GPS satellites with a local reference network," in ELMAR, 2011 Proceedings, Croatia, pages 321-324, IEEE, 2011.

[16] D. Odijk, P. J. G. Teunissen, and B. Zhang, "Single-frequency integer ambiguity resolution enabled GPS precise point positioning," J. Surv. Eng., vol. 138, no. 4, pages 193-202, 2012.

[17] X. Li and X. Zhang, "Improving the estimation of uncalibrated fractional phase offsets for PPP ambiguity resolution," J. Navig., vol. 65, no. 03, pages 513-529, 2012.

[18] X. Zhang, P. Li, and F. Guo, "Ambiguity resolution in precise point positioning with hourly data for global single receiver," Adv. Space Res., vol. 51, no. 1, pages 153 - 161, 2013.

[19] D. Odijk, P. J. G. Teunissen, and A. Khodabandeh, "Single-Frequency PPP-RTK: Theory and Experimental Results," IAG Symp, vol. 139, pages 167-173, 2014.

[20] X. Li, X. Zhang, and M. Ge, "Regional reference network augmented precise point positioning for instantaneous ambiguity resolution," J. Geod., vol. 85, no. 3, pages 151-158, 2011. 
[21] P. J. G. Teunissen, "A-PPP: Array-aided Precise Point Positioning with Global Navigation Satellite Systems," IEEE Trans. Signal Process, vol. 60, no. 6, pages 2870-2881, 2012.

[22] C. R. Rao, Linear Statistical Inference and its Applications. John Wiley \& Sons, New York, 1973.

[23] J. R. Magnus and H. Neudecker, Matrix Differential Calculus with Applications in Statistics and Econometrics. John Wiley \& Sons, New York, third ed., 2007.

[24] B. Hofmann-Wellenhof, H. Lichtenegger, and E. Wasle, GNSS: Global Navigation Satellite Systems: GPS, Glonass, Galileo, and More. Springer, New York, 2008.

[25] P. J. G. Teunissen, "Generalized inverses, adjustment, the datum problem and S-transformations," in Optimization and Design of Geodetic Networks (E. W. Grafarend and F. Sanso, eds.), pages 11-55, Springer, Berlin, 1985.

[26] P. J. de Jonge, A processing strategy for the application of the GPS in networks. PhD thesis, Delft University of Technology, Publication on Geodesy, 46, Netherlands Geodetic Commission, Delft, 1998.

[27] T. B. Andreasson and L. Engman, "Multipath at the SWEPOS stations," Evaluation of Eccsorb, a microwave absorbing material, Reports in Geodesy and Geographical Information Systems, vol. 2, 1999.

[28] A. Leick, GPS Satellite Surveying. John Wiley \& Sons, third ed., 2004.

[29] H. J. Euler and C. C. Goad, "On optimal filtering of GPS dual frequency observations without using orbit information," J. Geod., vol. 65, no. 2, pages 130-143, 1991.

[30] P. J. G. Teunissen, "A canonical theory for short GPS baselines. Part II: The ambiguity precision and correlation," J. Geod., vol. 71, pages 389-401, 1997. 